\title{
The challenge of many logics: a new approach to evaluating the role of ideology in Quinean commitment
}

\author{
Jody Azzouni
}

Received: 5 February 2014 / Accepted: 3 January 2015 / Published online: 13 January 2015

(C) The Author(s) 2015. This article is published with open access at Springerlink.com

\begin{abstract}
Can Quine's criterion for ontological commitment be comparatively applied across different logics? If so, how? Cross-logical evaluations of discourses are central to contemporary philosophy of mathematics and metaphysics. The focus here is on the influential and important arguments of George Boolos and David Lewis that second-order logic and plural quantification don't incur additional ontological commitments over and above those incurred by first-order quantifiers. These arguments are challenged by the exhibition of a technical tool-the truncation-model construction of notational equivalents - that compares the ontological role and increased expressive strength of non-first-order ideology to first-order ideology.
\end{abstract}

\section{The ethics of ontological commitment}

Quine's (1953) ethics of ontological commitment comes down to a simple principle (one, however, with background assumptions as well as stages of application): if you have to say it exists, then you're committed to its existence. Quine specifies "saying it exists" this way. What it is, that commits you to an entity $e$ that is $\mathrm{C}$, is $\exists x \mathrm{C} x$ in a first-order (standard Tarskian objectual) interpreted language, where $\mathrm{C} x$ is an arbitrary well-formed formula of this language with at most $x$ free, where what you have to say is $\mathrm{D}$; and $\exists x \mathrm{C} x$ logically (first-order) follows from $\mathrm{D}$. This is Quine's criterion for the commitments of a discourse, D, or (for short) Quine's criterion. ${ }^{1}$

\footnotetext{
1 As has become customary, I'm sloppy about "use and mention" while acknowledging that sloppiness. I also breezily use the metaphor "say" and related words. The criterion applies to discourses; and these don't have to be said or uttered or anything like that to have ontological commitments. They do, however, have to be meaningful. I make something of this requirement shortly.
}

J. Azzouni $(\varangle)$

Tufts University, Medford, USA

e-mail: jodyazzouni@mindspring.com 
Ontological commitment is concerned with what we say in the vernacular, since we always begin with theory or discourse that occurs in the vernacular (in mathematics, in empirical science, or in ordinary life). According to the Quinean approach, however, we're not to take ourselves as definitively committed until the relevant bit of vernacular speech has been recharacterized in first-order terms. (No claim is being made that a first-order recharacterization of a bit of vernacular is straightforward, easy, or uncontroversial.) In any case, the Quinean needn't care how ontological commitment is apparently conveyed in the vernacular itself-metaphorically, implicitly, explicitly, contextually - it can even be that our methods of indicating ontological commitment in the vernacular are hopelessly muddled.

Instead, and with Quine's criterion, a clean first-order tool for recognizing ontological commitment is given. A connection to the original vernacular speech remains because the descriptive and inferential roles of the original speech are replicated by first-order rendition - although never fully. Examples are, say, some applied rigorousinformal mathematics or a characterization of the lineage of a kind of insect. Whatever ontological readings we thought we detected (or thought we didn't detect) in the original speech, however, are set aside. We take seriously only the ontological commitments that are revealed according to the criterion when it's applied to the first-order rendition.

One red herring involves “explanation." Some philosophers complain that Quine's criterion inappropriately favors singular terms over predicate expressions. Contrary to straightforward applications of Quine's criterion, these philosophers say, we must explain what it is that red houses, red roses, and red sunsets have in common, and doing that shows that a commitment to a property, attribute or trope $\ll$ redness $\gg$ arises by merely claiming (for example) that roses are red.

Quine (1953, p. 10) is dubious about the explanatory value of such posited entities; but, strictly speaking, whether they have any explanatory value is irrelevant to the status of his criterion. If such an explanation is required-contrary to what Quine thinks — and if it's required, in particular, to explain commonalities like the redness of red roses, red sunsets, and red houses, then quantification over these entities occurs in whatever theory (of properties, attributes, or tropes, say) we have in which that explanation is given. Such an explanation and theory isn't given by saying, "Roses are red," or even "Roses and sunsets are red." Quantification over these entities occurs, perhaps, in our theory of the semantics of these expressions, or in the metaphysics needed to explain why these sentences are true. Quine's criterion is neutral about their existence, as it should be. Specifically, we don't need to treat predicates as tacitly ontologically committing to accommodate the value of this kind of explanation. ${ }^{2}$

\section{Boolos on the ontological role of plural and higher-order ideology}

Quine's criterion treats all the other idioms of an interpreted first-order formalism as irrelevant to ontological commitment. That is, the predicates and logical connectives in a first-order language are irrelevant to ontology. This isn't a stipulation, as the discussion of predicates in the previous section already indicates. The thought is that an

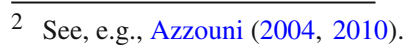


understanding of how predicates are involved in ontological commitment shows up in its proper place, a place where we theorize about the semantic/metaphysical role of predicates, and not when we simply use those predicates. So too with connectives. Consider, for example, the connectives in a bit of doctrine- "John is running and Sarah is jumping," or "John is running only if Sarah is jumping." Ontological commitments aren't taken to go beyond those sentences, of the form $\exists x \mathrm{C} x$, that follow from the first-order rendition of this doctrine. What guarantees this is the truth-functional transparency of first-order logic.

This point about first-order formalisms is broadly (although by no means universally) accepted. Disputes emerge when attempting to transplant Quine's criterion to other formalisms. In particular, the most heated debates ("heat," here, measured in the strange unit of numbers of articles and books dedicated to the topic) have arisen over plural quantification and monadic second-order quantification.

Some philosophers think that ideology (at least outside the first-order context) can contribute to ontology as well. ${ }^{3}$ The debate is: how? And relatedly: how do we tell what the ontological role of a particular bit of ideology is? Quine uses the following secondary criterion for evaluating the ontological-commitment role of the ideology of an alternative logic: render that ideology in an interpreted first-order formalism, and see what else (in first-order terms) it's committed to. ${ }^{4}$

Two important points. First, what I'm calling "the secondary criterion" only extends Quine's view of ontological commitment in the vernacular to non-first-order ideology. The vernacular, he thinks, is no place to directly evaluate ontological commitments. Quine takes the same view of non-first-order discourses.

Second, one can ask: Why stress interpreted first-order formalisms? Answer: Because only sentences that say things have ontological commitments. Couple this truism with Quine's assumption that quantifier commitments are ontologically relevant only when those quantifiers are objectual, and Tarskian models (or something equivalent) emerge as the mechanism of interpretation.

A proponent of the strict Quinean criterion is committed both to (i) Quine's criterion, and (ii) Quine's secondary criterion. Notice that this individuates Quine's criterionto begin with-as something applied to discourses in the vernacular. (This is the only application Quine discusses in his (1953)). I'm therefore treating Quine's secondary criterion as a broadening of the first criterion to encompass discourses in the language of one or another alternative logic. 5

\footnotetext{
3 E.g., Shapiro (1997), p. 54.

4 Quine (1970, p. 66), using this first-order-rendition methodology, describes higher-order logic as "just set theory in sheep's clothing."

In an earlier version of this paper, I used "translate" instead of "render," but that's misleading as a referee has made clear to me. As with the vernacular, Quine isn't interested in meaning- or (full) truth-conditionpreservation, or other niceties that one might expect from a "translation." This is one of the objections Boolos raises to Quine's first-order rendering of higher-order logic, as I'll indicate later in this section. Rendering is an optimal (but not perfect) first-order capture of the various expressive/logical properties of the non-first-order target.

5 A referee has suggested a different way to characterize Quine's criterion: No need for first-order rendition; just look at what's quantified over in the first-order fragment of the discourse in question. Then the criterion applies straightaway both to the vernacular and to discourses in certain alternative logical systems.
} 
The strict Quinean criterion, to put it mildly, hasn't wide appeal. Philosophers, among them, Boolos (1984, 1985); Field (1980, 1989); Lewis (1991), and van Inwagen (1990), embrace their favorite non-first-order formalisms, but then deny that theories in these formalisms incur the ontological commitments that first-order renderings of them have.

Noting that this secondary criterion isn't justified by virtue of accepting Quine's criterion, Boolos (1985, p. 78) writes:

We ought to recall that logicians have devoted attention to quantifiers other than the usual for all $x$ and for some $x$. Among these less familiar quantifiers, which cannot be defined by means of the apparatus of first-order logic, are for most $x$, for infinitely many $x$, for uncountably many $x$, and for at least as many $x$ as there are objects (in the domain). To claim that a statement to the effect that there are infinitely many objects of a certain kind, made with the aid of the quantifier for infinitely many $x$, implies the existence (on the customary acceptation of those words) of an (infinite) class solely on the ground that the only way to utilize more familiar logical vocabulary to eliminate the unfamiliar quantifier is to employ a quantifier ranging over classes is to invite the response: what makes first-order logic the touchstone by which the ontological or existential commitments of these statements are to be assessed? The statements do not appear to commit us to classes; why believe that it is their translation into the notation of first-order logic augmented with variables ranging over classes that determines what they are actually committed to ${ }^{6}$

Boolos is not challenging Quine's criterion. His arguments are meant to support a conservative version of Quine's criterion, at least with respect to plural-quantification formalisms and higher-order logic: treat only the first-order quantifiers as indicating ontological commitment even when they occur in a formalism accompanied by other non-first-order idioms. ${ }^{7}$ He is challenging Quine's offered method of evaluating the ontological commitments of theories utilizing non-first-order idioms: rendering those theories in a pure (interpreted) first-order formalism; he's challenging Quine's strict criterion.

\footnotetext{
Footnote 5 continued

But this isn't a good way to initially think about Quine's criterion for at least two reasons. First, it doesn't sensibly apply to the vernacular at all since, without a well-understood empirical characterization of the syntax of the vernacular (which we lack), the first-order fragment isn't well defined. Second, it's not prima facie suitable for non-first-order logics, since Quine's criterion is supposed to evaluate the ontological commitments of entire discourses, and the first order fragment of a discourse doesn't indicate what the rest of the discourse is doing ontologically. A supplementary argument that the other idioms of the discourse are ontologically innocent is needed. As I'll show later, arguments to establish what amounts to this suggestionagainst Quine's secondary criterion—are offered by Boolos and other philosophers.

6 See Field (1980), pp. 94-5, where the same argument uses instead the There is finitely many quantifier.

7 Boolos (1984, p. 72) writes, when speaking of discourse with both first- and second-order quantifiers, that: "Ontological commitment is carried by our first-order quantifiers; a second-order quantifier needn't be taken to be a kind of first-order quantifier in disguise, having items of a special kind, collections, in its range." On Boolos' view: $\exists X \exists x X x$ is committed only to there being a (first-order) entity-not to there being a second-order entity that holds of the first-order entity. This view contrasts with Shapiro (1985), for example, who accepts that the second-order existential quantifier is committing just as the first-order one is.
} 
In any case (as Boolos also points out), the Quinean suggestion of evaluating ontological commitments of theories (or non-first-order formalisms themselves) by first-order rendition looks misguided (and not merely unjustified). To take higherorder logic as an example, the rendition in first-order set theory that Quine urges, replacing " $\mathrm{F} x$ " with " $x \in \alpha$," and quantifying on the set-theoretic variable " $\alpha$ " rather than on the predicate "F," doesn't preserve the important and apparently relevant metalogical properties of the original formalism. First off, higher-order logic looks genuinely like logic - in particular it's an uninterpreted formalism requiring models to make its sentences (fully) meaningful. Like first-order logic, it introduces a division between logical constants and other terms. As a result, one studies the models (the interpretations) of theories in the language of higher-order logic just as one studies the models of theories in the language of first-order logic. One proves, for example, that completeness and Löweinheim-Skolem fail, although both are true of first-order logic. As Boolos (1975, pp. 39-40) stresses, higher-order logiclooking rather like a logic, looking rather like first-order logic in particular-has validities such as $\exists X \forall x X x$, but these aren't rendered as truths of set theory, say ZFC.

First-order set theory-to press these differences further-is an entirely different beast. It's a fully interpreted formalism - with sentences-not a system of (partially uninterpreted) formulas. It has nonstandard models, of course-but this is hardly the same thing as uninterpreted theories of higher-order logic needing models to supply them with interpretations. And, anyway, the previous observations show that they don't share the same models - not by a long shot. It's simply not obvious, therefore, that the ontological commitments of interpreted higher-order logics should be evaluated by those of a first-order set theory, as Quine suggests.

The problem is quite general, applying to all the examples of non-first-order ideology that Quine would apply his rendition method to, some of which Boolos mentions in the quotation I gave earlier. But rejecting Quine's secondary criterion leaves us without a general and systematic method to compare the ontological commitments of theories across formalisms. ${ }^{8}$ The result is that Boolos (and other philosophers) fall back on natural-language intuitions about whether natural-language analogues of non-first-order ideology introduce ontological commitments, as well as the occasional argument (as we'll see in Sect. 6). Thus, debates about whether plural quantification introduces ontological commitments over and above first-order logic are unresolvable precisely because there is currently no way of systematically comparing ontological commitments across logics - in particular, between first-order logic and non-first-order competitors - other than Quine's contested (and widely rejected) first-order rendition technique. These debates, furthermore, are given extra philosophical angst because plural quantifiers (in particular) have been widely adopted by a generation or more of mainstream metaphysicians to motivate the cogency of metaphysical positions that aren't possible in first-order terms (because they're undercut by first-order indispensability arguments). ${ }^{9}$ As I've just mentioned, without a techni-

\footnotetext{
8 We would have such a method if the suggestion of footnote 5 could be made to fly. But it can't.

9 Two early influential examples: Lewis 1991 and van Inwagen 1990. Both philosophers (Lewis explicitly and van Inwagen implicitly_via Lewis's authority) accept Boolos' claim that plural quantification
} 
cally justified method of comparing ontological commitments across formalisms, we have only the tool of natural-language ontological-commitment intuitions about the sentences in question; and Boolos wields this tool with admirable rhetorical power, when he writes (1984, p. 72), speaking of some Cheerios in a bowl:

There are, of course, quite a lot of Cheerios in the bowl, well over two hundred of them. But is there, in addition to the Cheerios, also a set of them all? And what about the $>10^{60}$ subsets of that set? (And don't forget the sets of sets of Cheerios in the bowl.) It is haywire to think that when you have some Cheerios, you are eating a set-what you're doing is: eating THE CHEERIOS. Maybe there are some reasons for thinking there is such a set-there are, after all, $>10^{60}$ ways to divide the Cheerios into two portions-but it doesn't follow just from the fact that there are some Cheerios in the bowl that, as some who theorize about the semantics of plural would have it, there is also a set of them all.

This is, essentially, the same point Black (1971, p. 615) makes some years earlier. Black first quotes Halmos (1960, p. 1): "A pack of wolves, a bunch of grapes, or a flock of pigeons are all examples of sets of things," and then he adds sarcastically: "It ought then to make sense, at least sometimes, to speak of being pursued by a set, or eating a set, or putting a set to flight."

Although this is compelling rhetoric, it's still just rhetoric-based ultimately only on the sheer intuitive oddity of saying things in the vernacular like "I'm eating a set," "I'm chasing a set," or "I'm scared of that (evil) set." We need definitive tools to adjudicate this debate.

\section{Equivalence}

The point of this paper is to justify a different method of evaluating the ontologicalcommitment role of non-first-order ideology, a method without the drawbacks that Boolos and others have exposed in Quine's first-order-rendition method. Here's how I'll present this method. First, in this section, I'll present Equivalence, ${ }^{10}$ the principle that notationally-equivalent logics have the same ontological commitments. I'll then describe, in Sect. 4, a disagreement in the literature about the legitimacy of the additional expressive strength of theories in higher-order logics over first-order theories, and I'll illustrate the technical details of the truncation-model method of constructing notationally-equivalent logics by applying it to the logics involved in this debate. I'll then turn to plural quantification in Sect. 5 .

Consider logics $L$ and $L^{*}$, and model-theoretic semantics, $M$ and $M^{*}$ respectively, for those logics. It's pretty standard to understand a semantics (a Tarskian-style semantics, say) as giving interpretations for the sentences couched in the formalism of that

Footnote 9 continued

introduces no additional ontological commitments above and beyond singular first-order quantifiers. To say, for example, "There are some apples on the table," or "There are some molecules arranged chairlike," is to be committed (respectively) to individual apples or individual molecules, and nothing more. I discuss this in Sect. 5.

10 Thanks to a referee for this term. 
logic_as I've been doing all along. Accepting this, we can describe $L / M$ and $L * / M^{*}$ as notational equivalents if there is a mapping, $\mathrm{I}^{M L}$ that's one-to-one between the models of $M$ and $M^{*}$, and one-to-one between the sentences of $L$ and $L^{*}$, so that if a sentence $S$ of $L$ is true-in a model $m$, then its image $\mathrm{I}^{M L}(S)$ is true-in $\mathrm{I}^{M L}(m)$.

Notice that, given appropriate proof-theoretic choices (ones compatible with I ${ }^{M L}$,s mapping of the sentences), $\mathrm{I}^{M L}$ preserves all the significant metalogical properties, for example, whether the interplay between the $L s$ and $M$ s induces essential incompleteness, details about expressive strength, and so on. The proponent of the syntactic ethics grounding Quine's criterion should accept (therefore) that $L / M$ and $L * / M^{*}$ share their ontological commitments-whatever they are. The reason is that the ontological commitments of a theory (in a logic) should supervene on the relationship of that logic to its semantics. Quine, specifically, repeatedly stresses that his first-order criterion is intrinsically related to the semantics of first-order logic being objectual. ${ }^{11}$ Call Equivalence the principle that any two logics isomorphic in the above sense are equivalent in their ontology-inducing capacities.

Equivalence allows an in-principle tool to measure exactly what the ideology of a non-first-order logic introduces over and above a first-order formalism-and, in particular, a tool independent of the puzzling issues surrounding first-order rendition. Start with some non-first-order $L / M$, and construct a notational variant of it, $L^{*} / M^{*}$, and an appropriate $\mathrm{I}^{M L}$, by doing one or more of the following to an initially-given first-order formalism: (i) introducing additional logical constants into the formalism, (ii) restricting the admissible models, (iii) restricting the admissible expressions of the language of that formalism. Whatever additional ontological commitments $L * / M^{*}$ induces beyond those of the first-order formalism it was constructed from can be imputed to that $L / M$ by virtue of its notational equivalence to $L * / M^{*}$. More generally, all the ways that $L / M$ differs from first-order formalisms in expressive capacities can be evaluated by the construction, via (i)-(iii), of $L^{*} / M^{*}$.

Although Quine's rendition method yields first-order renditions, notice that Equivalence doesn't. The result of an application of Equivalence, a first-order formalism supplemented with logical constants that's in addition restricted in its language and/or model theory - is not first-order. To repeat: Its differences from a first-order formalism with standard models can be precisely measured by its construction via (i)-(iii).

Equivalence, perhaps, allows an Alston (1958)-style rejoinder: $L / M$ hasn't the additional "now-exposed" ontological commitments of $L^{*} / M^{*}$; rather, $L^{*} / M^{*}$ has the narrower-construed ontological commitments of $L / M$. After all, by assumption, the formalisms are notational equivalents. The response: Recall the rules of the game. This paper attacks those who accept Quine's criterion when applied to first-order logics, but who reject Quine's strict criterion (as Boolos, Lewis, van Inwagen, and many others do): who think accepting Quine's criterion is compatible with it being soundly applicable to non-first-order formalisms, such as higher-order logics, pluralquantification formalisms, etc. ${ }^{12}$

\footnotetext{
11 This commitment is made polemically by him to officially contrast his attitude to substitutional quantification, where "all ontological bets are off." See, e.g., Quine (1974). In this discussion, he commits himself to Tarskian model theory, in addition to doing so in other relevant contexts, e.g., Quine (1970).

12 My thanks for an anonymous referee for urging a response to this Alston-style rejoinder.
} 
Recap. I first characterized Quine's strict criterion this way: apply it to an interpreted first-order formalism or first-order render your target formalism, and identify its commitments with those of the rendition. The Boolosian rejoinder is that so-rendering demotes non-first-order logical idioms to interpreted non-logical first-order ideology, and that distorts their philosophically-relevant properties-notably their metalogical properties. The strict-criterion proponent retorts: I accept your objection. But now I demand an application of Equivalence. For only by determining how your non-firstorder logical formalism goes beyond first-order resources can we evaluate whether or not additional ontology is induced.

I treat Equivalence as a friendly amendment to the strict Quinean criterion: Equivalence replaces "rendition in an interpreted first-order formalism." I now turn to applying Equivalence to monadic second-order logic.

\section{Measuring the role of ideology in ontology: the model-truncation recipe and its application to monadic second-order logic}

A (still-living) topic in philosophy of mathematics is referential access to mathematical entities. A version of a worry about this is that first-order theories (because of Löweinheim-Skolem theorems) have non-standard models of varying cardinalities. One is concerned, naturally enough, that therefore it isn't possible to refer to and only to the intended model by means of a first-order number theory (however axiomatically rich) because any such theory has not just an intended model but numerous other ones of arbitrary cardinality (and far richer structure). This problem can be tamed-up to model-isomorphism-by using instead monadic second-order logic with standard (nonHenkin) semantics. A debate arises, therefore, about whether deserting the firstorder context legitimately secures reference (modulo isomorphism) to the standard model of arithmetic. Shapiro (1985), for example, claims that second-order logical theories don't illegitimately presuppose a greater referential grip on pertinent mathematical objects than appropriate, although this $i s$ true for theories couched in $\omega$-logic. ${ }^{13}$

First-order proponents were concerned with the following issue: the additional resources that second-order logic seems to have to rule out non-standard model aren't legitimate. I won't revisit this debate, ${ }^{14}$ but instead note two points. First, the issue isn't—strictly—about ontological commitments; it's about expressive strength. Model-theoretic options that first-order theories can't descriptively rule out are apparently ruled out by theories couched in higher-order logics. The debate-question is:

$13 \omega$-logic supplements standard logic with an additional quantifier that ranges over the natural numbers. Shapiro (1985, p. 733) writes that $\omega$-languages "cannot be used to show, illustrate, or characterize how the natural number structure is itself understood, grasped, or communicated," since "they assume or presuppose the natural numbers." At that time, Shapiro claimed that he took second-order logic not to exhibit this vice of assuming or presupposing the natural numbers: he took its semantics to be legitimately adequate to axiomatizing arithmetic, real and complex analysis, etc., in contrast to the semantics of first-order languages (Shapiro 1985, pp. 714-715). He has subsequently denied claiming this, however, in Shapiro (1999), specifically p. 58. Post-1985 Shapiro, in any case, has weakened his brief for the value of higher-order logics, recently writing (Shapiro 2012, p. 305) that "second-order languages, with standard model-theoretic semantics, have important roles to play in foundational studies."

14 See Azzouni (1994). See Shapiro (1999), for further discussion. 
what is it about theories couched in second-order logic that enables this? Second, my present aim, in any case, is to illustrate a recipe for measuring differences in formal theories couched in different logics. Along the way, comparison techniques for ontological commitments are introduced too.

So, consider a second-order monadic predicate calculus containing $n$-place predicate symbols; but where second-order quantification is only one-place. And presume a standard semantics (all the subsets of the domain are implicitly ranged over by the second-order quantifiers). More specifically, consider a two-sorted-variable secondorder language: $\forall x, \exists x, \forall X, \exists X$, with individual constants: $\mathrm{a}, \mathrm{b}, \mathrm{c}, \ldots$, individual variables, $x, y, z, \ldots$, various $n$-place predicate constants: $\mathrm{P}, \mathrm{Q}, \mathrm{R}, \ldots$, and (one-place) predicate variables, $X, Y, Z, \ldots$. Accompany this with a standard second-order (nonHenkin) semantics, in particular, with standard models $A_{\mathrm{s}}$. That's $L / M$.

I now construct $L * / M^{*}$ from a set of first-order languages with standard Tarskian model theory. I call the forthcoming logic $(2 \mathrm{~m})$ truncated logic. ${ }^{15}$ To construct it, we restrict both the admissible models and the admissible sentences of an initial firstorder language. The vocabulary of the language we start from has standard quantifiers, $\forall x, \exists x$, individual constants: $\mathrm{a}, \mathrm{b}, \mathrm{c}, \ldots$, individual variables, $x, y, z, \ldots, n$-place predicate constants: $\mathrm{P}, \mathrm{Q}, \mathrm{R}, \ldots$... First, introduce two distinguished predicates, "S" and "E," deemed additional logical constants. That is, they are reinterpreted across models in constrained ways. Consider the set of sentences SS that's constructed using the standard formation rules from this vocabulary. That's not the set of sentences of $L^{*} . L^{*}$, instead, is the image of the mapping $\mathrm{I}^{L}$ of the sentences of $L$ into SS. I'll indicate details about $\mathrm{I}^{L}$ once other aspects of the to-be-constructed $L * / M^{*}$ are given.

The model theory $M *$ contains only certain highly-structured first-order models $m *$. First, the domain $d_{m^{*}}$ of any such model $m^{*}$ is bifurcated: its domain $d_{m^{*}}=d \cup \mathcal{P}$ d. An interpretation (a $(2 m)$ truncated model $m^{*}$ ) of SS (the first-order language $+\{\mathrm{E}, \mathrm{S}\}$ ) in any $(2 m)$ truncated domain is the same as for standard first-order theories, except: (i) in each model, the one-place predicate " $\mathrm{S}$ " is mapped to $\mathcal{P} \mathrm{d}$, the one-place predicate "E" is mapped to $\{(a, b) \mid a \in b \& a \in \mathrm{d} \& b \in \mathcal{P} \mathrm{d}\}$, and (ii) the extensions and antiextensions of the other predicates and relations are restricted to d. Importantly, this includes identity. Call these $(2 m)$ truncated models.

To proceed, I must present the above-mentioned mapping $\mathrm{I}^{L}$ of the sentences of $L$ into SS. This mapping treats this version of second-order logic as a two-sorted first-order logic, and relativizes the two sets of quantifiers to one. ${ }^{16}$ Specifically, occurrences of higher-order quantifiers $(\forall X) \ldots X \ldots,(\exists X) \ldots X \ldots$, are replaced by $(\forall x)(\mathrm{S} x \rightarrow \ldots x \ldots)$ and $(\exists x)(\mathrm{S} x \& \ldots x \ldots)$, respectively. Occurrences of first-order quantifiers $(\forall x) \ldots x \ldots,(\exists x) \ldots x \ldots$, are replaced by $(\forall x)(\neg \mathrm{S} x \rightarrow \ldots x \ldots)$, and $(\exists x)(\neg \mathrm{S} x \& \ldots x \ldots)$, respectively. (A corresponding mapping is in place for the individual constants.) Lastly, all instances of predication, where a higher-order variable appears, $Y \mathrm{t}$, are replaced with Et $Y$. $\mathrm{I}^{L}$ is a mapping of $L$ properly into SS (many sentences are left out). $L^{*}$ is the image of $\mathrm{I}^{L}(L)$.

\footnotetext{
15 This is what I called it in Azzouni (1994), § 3, where I first gave this construction. I called it this because (as I will subsequently show) this (restricted) language, as it's interpreted by the introduced model theory, is a notational equivalent of second-order monadic logic with standard semantics.

16 See van Benthem and Doets (1983).
} 
Notice that, along with $\mathrm{I}^{L}$, I've also implicitly given a 1-1 mapping of the (standard) models of monadic second-order logic to the models of $(2 \mathrm{~m})$ truncated model theory. Any model $m$ of monadic second-order logic with a domain $e$ is mapped to that model $m^{*}$ of $(2 m)$ truncated model theory induced by letting $\mathrm{d}=e$, and holding fixed the interpretations of the nonlogical vocabulary of $L$ in $e .{ }^{17}$ So I've exhibited the needed $\mathrm{I}^{M L}$.

The interesting (and relevant) metalogical properties, e.g., versions of completeness, compactness, and Löweinheim-Skolem, arise from the interplay of the model theory and the language of the logic under study. It's easy to show monadic second-order logic plus standard semantics has the same metalogical properties that $(2 m)$ truncated logic has. Specifically, compactness, Löweinheim-Skolem, and completeness fail, and the two logics have identical capacities to characterize infinite structures. They are notational variants in the required sense. ${ }^{18}$

Important to evaluating how monadic second-order logic goes beyond first-order resources is seeing what can be said in both monadic second-order logic and $(2 m)$ truncated logic, and as importantly, seeing what can't be said in both monadic second-order logic and $(2 m)$ truncated logic. First, two examples of the sayable.

Consider the second-order induction schema for PA. ${ }^{19}$

$$
\forall X([X 0 \& \forall x(X x \rightarrow X \mathrm{~s} x)] \rightarrow \forall x X x) .
$$

This corresponds to

$$
\forall x(\mathrm{~S} x \rightarrow([\neg \mathrm{S} 0 \& \mathrm{E} 0 x \& \forall y(\neg \mathrm{S} y \rightarrow(\mathrm{E} y x \rightarrow \mathrm{Es} y x))] \rightarrow \forall \mathrm{y}(\neg \mathrm{S} y \rightarrow \mathrm{E} y x))) .
$$

Consider next a second-order formulation of a relation $\mathrm{R}$ being well founded:

$$
\forall X[\exists x(X x \rightarrow \exists x(X x \& \forall y(X y \rightarrow \neg \mathrm{R} x y)))] .
$$

This corresponds to

$\forall z(\mathrm{~S} z \rightarrow[\exists x(\neg \mathrm{S} x \& \mathrm{E} x z \rightarrow \exists x(\neg \mathrm{S} x \& \mathrm{E} x z \& \forall y(\neg \mathrm{S} y \rightarrow(\mathrm{E} y z \rightarrow \neg \mathrm{R} y x))))])$.

As I mentioned, it's equally significant that certain things can't be said, things that are least syntactically sayable in the sense that they are sentences of SS but not of $L^{*}$.

\footnotetext{
17 Two small technical matters about the definition of true-in for $L^{*} / M^{*}$. First, the truth-clauses for model $m^{*}$ take account of the interpretation of the items of $\mathcal{P} \mathrm{d}$ as subsets of $\mathrm{d}$. This enables the $(2 \mathrm{~m})$ truncationcapture of monadic second-order quantifier-instantiation to predicate constants. Second, the truth-clauses are defined for all sentences in SS, and the values of sentences outside the image of $\mathrm{I}^{L}$ are discarded. It's shown by a standard induction that if a sentence $S$ of $L$ is true-in a model $m$ of $M$, then $\mathrm{I}^{M L}(S)$ is true-in $\mathrm{I}^{M L}(m)$.

18 Meta-Proof sketch: Pick your favorite metalogical property of monadic second-order logic. Its proof turns on the interplay of the language/particular-proof-theory of monadic second-order logic $L$ with its model-theory $M$. Using $\mathrm{I}^{M L}$, every step of the proof can be replicated. Notice in particular, that any proof-procedure applicable to $L$ corresponds to a proof-procedure applicable to $L^{*}$ via the mapping ${ }^{L}$.

19 A function symbol appears in the two formulas to follow. It can be treated as language defined from predicates or the earlier characterizations of $L$ and $L^{*}$ can be modified to include functions.
} 
The unsayable graphically illustrates the ways monadic higher-order logic is restricted in its expressive powers. Here are two examples. ${ }^{20}$

$$
\begin{gathered}
(\forall x)(\mathrm{S} x \vee \neg \mathrm{S} x) \\
(\forall x)(\forall y)(\mathrm{S} x \& \mathrm{~S} y \rightarrow(x=y \leftrightarrow(\forall z)(\mathrm{E} z x \leftrightarrow \mathrm{E} z y))) .
\end{gathered}
$$

The first statement asserts the logical truth, in $(2 \mathrm{~m})$ truncated logic, that everything is either a set or not. The second statement asserts the extensionality property that holds of the items in $\mathcal{P}$. The reason for these bits of censorship - and others like them-isn't far to seek. Monadic second-order logic is severely restricted in what it can say about the items it second-order quantifies over (and thus, correspondingly, $(2 \mathrm{~m})$ truncated logic is similarly gagged). This is indicated in monadic second-order logic by what's sayable — as it were - about these things involving only second-order quantifiers. Specifically, the $n$-place predicates are (implicitly) barred from ranging over anything other than the members (or instances) of what the second-order quantifiers range over. This is explicitly captured in $(2 m)$ truncated logic by the corresponding restriction that the extensions and anti-extensions of the $n$-place predicates, including identity, are constrained to d, the individual constants hold only of items in d, and the $n$-place predicates and individual constants occur only within the scope of " $\neg \mathrm{S}$."

These expressive limitations are due, however, to monadic second-order logic being monadic and second-order. My purpose in stressing them now has largely been to illustrate the power and flexibility of the truncation-model approach to non-first-order formalisms - and in particular, the adaptability of the methods (i)-(iii), of constructing notational variants, to non-first-order peculiarities. I'll mostly leave aside mentioning expressive limitations in the polemical discussion (about ontological commitments and expressive strength) that follows.

Let's turn, therefore, to how we should regard the supplementations to first-order logic that yield $(2 m)$ truncated logic. First-order logic allows arbitrary domainsexcept for the exclusion of the empty domain. Any model of $(2 m)$ truncated theory, by contrast, is not arbitrary: the domains of these models are bifurcated into "urlements" and all the subsets of the set of urlements. ${ }^{22}$

Directly related to the increased expressive strength of $(2 \mathrm{~m})$ truncated logic (and consequently to the increased expressive strength of second-order monadic logic) is (i) the logical constant " $\mathrm{S}$ " distinguishing between set-like items and urlement-like items in any model of $(2 \mathrm{~m})$ truncated logic, and (ii) the logical constant " $E$ " that characterizes "is a member of" relations between the urlement-like elements and the

\footnotetext{
20 My thanks to a referee for stressing this neat example.

21 Notice that these last remarks show that we can characterize the language $L *$ directly (and independently) of the mapping $\mathrm{I}^{L}(L)$. This is important to notice (as a referee has stressed to me) because otherwise another version of the Alston argument can arise: namely $L^{*}$ is parasitic on $L$, and so inherits $L$ 's ontological commitments. Because second-order quantification is innocent, $L^{*}$ doesn't convict $L$ of any commitments beyond those carried by $L$ 's first-order quantifiers.

22 I use "subset" (and, earlier, "set") because these are easy terms to reach for in these contexts. I'll raise considerations that challenge strictly so-interpreting this terminology, later in this section and in the next one.
} 
set-like elements. In using phrases like "urelement-like" and "set-like" I'm indicating that these relationships are relative to the model: They don't target whatever settheoretical relations occur among the items in $\mathrm{d}$ (which are urelements according to the model theory).

Some are tempted, nevertheless, to describe $(2 m)$ truncated logic as "set theory." If "theory" in "set theory" is meant seriously, this should be resisted. We can say very little about these sets. This is perhaps already directly noticeable by looking at monadic second-order logic; but it's completely transparent in $(2 m)$ truncated logic-if only because of the many sentences of SS excluded from $L^{*}$. Indeed, monadic secondorder logic —all on its own-looks like a natural generalization of first-order logic: we quantify into predicate positions just as we first-order quantify into individual-constant positions. But its notational equivalent, $(2 \mathrm{~m})$ truncated logic, reveals the artificialities. We quantify over things rather like sets, and not, say, like proper classes. But, nevertheless, there are no predicates that hold of them. Totally weird. ${ }^{23}$

It's clear, despite the foregoing, why set-theoretic phenomena intrude into the semantic properties of $(2 \mathrm{~m})$ truncated logic, and thus correspondingly into the semantic properties of monadic second-order logic. In any case, and very importantly, the additional logical constants introduce expressive strength simply because it's now possible to eliminate models altogether that would be available otherwise.

The roles of "E" and "S" are explicit in $(2 \mathrm{~m})$ truncated logic, and so it would be hard to argue that shifting from first-order logic to $(2 \mathrm{~m})$ truncated logic is innocent, in the sense (to paraphrase Shapiro (1985, p. 733)) that $(2 m)$ truncated logic doesn't "assume or presuppose the natural numbers." The notation presupposes something a lot stronger. What makes it easy to think that monadic second-order logic doesn't assume or presuppose something (equivalent or superior in strength to) the natural numbers? One thing is that the roles of " $\mathrm{E}$ " and " $\mathrm{S}$ " are hidden in monadic second-order logic. In first-order logic, the concatenation of a (one-place) predicate "P" with a constant "a," as in "Pa," needn't be taken to contain an (implicit) representation of some sort of membership relation. But when we quantify (standardly) into the predicate position, as we do in monadic second-order logic, this is how syntactic concatenation of individual terms with predicate terms must be understood (as the notational equivalence of $(2 m)$ truncated logic with monadic second-order logic shows). Further, and this is striking (or ought to be), syntactic concatenation of one-place predicates with constants in the monadic second-order context can't be reinterpreted across modelsas the notational equivalence to $(2 \mathrm{~m})$ truncated logic also indicates-it's therefore an (implicit) logical constant. ${ }^{24}$

\footnotetext{
23 Don't trivialize the question whether $(2 \mathrm{~m})$ truncation logic is a theory of sets-and therefore, correspondingly, the question whether monadic second-order logic is-by thinking that there are all sorts of set theories, including quite weak ones, and so, of course it is. There are two issues, first, whether what can be said about sets in these languages is enough to call the result "set theory," the issue just explored. But, second, there is whether the relations that the items in $\mathcal{P}$ d bear to the items in $\mathrm{d}$ are sufficient to call the items in $\mathcal{P d}$ "sets." I explore this briefly in the next section. My thanks to a referee for prompting this footnote.

24 Notice its role shows up in two ways in $(2 m)$ truncated logic. First, predication is explicitly replaced by $\mathrm{E}$, when a second-order variable is involved, but second, in any $(2 \mathrm{~m})$ truncated model, predicate constants are linked to specific items in $\mathcal{P}$ d by the definition of true-in. (See footnote 17.)
} 
A second way that second-order monadic logic misleads. Second-order quantifiers under the standard interpretation not only range over all the subsets relativized to the domain, but also only over such objects. This is enabled by two peculiar facts about second-order quantifiers and their model theory: (i) they quantify into predicate places, and (ii) their range is only implicitly given-that is, what they range over appears nowhere in the domain. But when we isomorphically map monadic secondorder logic over to $(2 m)$ truncated first-order logic, everything (and "something" too) becomes terminologically transparent. When the $(2 m)$ truncated first-order quantifiers mimic the second-order quantifiers under the standard interpretation, they are explicitly fixed by logical-constant predicates that hold of part of the domain (in every $(2 \mathrm{~m})$ truncated model). The notation (and this is made explicit in $(2 \mathrm{~m})$ truncated logic) has the expressive capacity to focus on part of what there is in a domain to the exclusion of other things there are in that domain.

Let's return to how the natural-number structure (in Shapiro's (1985) words) is itself "understood, grasped, or communicated." Compare first-order Peano arithmetic with the $(2 m)$ truncated-logic version of monadic second-order Peano arithmetic (hereafter ( $2 \mathrm{~m}$ )Peano arithmetic). Relevant to the comparison isn't merely, from the point of view of the $(2 \mathrm{~m})$ truncated models, that in addition to the numbers that are quantified over, the members of the powerset of those numbers are also quantified over. Far more important is that the membership relations between the numbers and the items containing them are preserved (by the logical constancy of "E" and "S") across all possible $(2 m)$ truncated models of $(2 m)$ Peano arithmetic. It's this, of course, that gives $(2 \mathrm{~m})$ Peano arithmetic (and therefore, via its notational equivalence, monadic secondorder Peano arithmetic) the expressive capacity to exclude the $(2 \mathrm{~m})$ truncated models that would correspond to the non-standard models of first-order Peano arithmetic. As a result, describing second-order Peano arithmetic as not inappropriately presupposing the natural numbers (contrary to $\omega$-logic) misses the point of the objection that secondorder Peano arithmetic has illegimately greater expressive powers. (It's a second red herring.) The focus should be on the introduced logical constants, and their legitimacy.

An easy conclusion: Only if someone thinks it's legitimate to: (i) restrict the arbitrary models of first-order logic as it's done in $(2 m)$ truncated logic, and (ii) stipulate additional logical strength by means of logical constants, like "E" and "S," should that someone think adopting monadic second-order logic offers a solution to the question of how the natural number structure is itself "understood, grasped, or communicated." 25

\footnotetext{
25 See Azzouni 1994, § 3, footnote 20, and surrounding material, for details on generalizing the truncationmodel method to full second-order logic. Similar techniques can be used on various generalized-quantifier logics that Boolos alludes to (in the quotation I've given). A technical question arises: How general is the model-truncation method? It seems quite general, actually, as already indicated. Consider, for example, a modal formalism with (classical language) possible-world semantics. The first step of the truncation-model recipe uses the truth-conditions of the modal formalism to capture that modal formalism in an interpreted first-order language. This yields a significant increase in ideology: not just " $\alpha$ is in world $\beta$ " but also new non-logical predicates (corresponding to the non-logical predicates in the original modal formalism) with additional places for worlds. The second step transforms this new ideology into a set of logical constants corresponding to a restricted set of first-order models, as well as a restriction of the set of admissible sentences expressible in that ideology. (My thanks to one of the referees for asking about generalizations of the model-truncation method.) Notice that a dramatic "uncovering of hidden ontology" doesn't really occur in the modal case as it does with higher-order logics. This is because the possible-world semantics of
} 


\section{An application of the model-truncation recipe to plural quantifiers}

Assume we have a theory of some sort, or (more vaguely and uncontroversially) a way of describing ordinary objects, such as furniture. We talk about chairs, for example, and describe some of their properties - they're artifacts for example, most of them have four legs, they're composed of atoms, etc. Call this (indeterminately large) set of statements $\mathrm{D}^{\mathrm{v}}$. We speak in the vernacular about these things $\left(\mathrm{D}^{\mathrm{v}}\right.$ is a set of statements of the vernacular), but on one or another implementation of the Quinean ontological strategy, we reframe $D^{v}$ as $D^{f}$ : the statements in the vernacular are reconstrued in one or another first-order logical framework. The ethics of Quinean commitment forces the following paraphrase condition: If $(\exists x) \mathrm{C}$ is a consequence of $\mathrm{D}^{\mathrm{f}}$, then by virtue of a commitment to the truth of the statements of $\mathrm{D}^{\mathrm{f}}$, a commitment to C-entities is incurred unless it's possible to reformulate $\mathrm{D}^{\mathrm{f}}$ as $\mathrm{D}^{\mathrm{f} *}$, where $(\exists x) \mathrm{C}$ isn't a consequence of $\mathrm{D}^{\mathrm{f} *}$, and where-for the purposes the discourse $\mathrm{D}^{\mathrm{v}}$ is put to- $\mathrm{D}^{\mathrm{f} *}$ is as $\operatorname{good}$ as $\mathrm{D}^{\mathrm{f}}$.

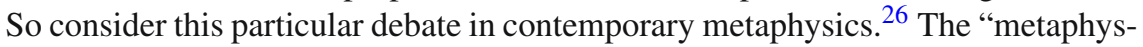
ical nihilist" claims that only metaphysical simples exist. A chair isn't a metaphysical simple, so the metaphysical nihilist denies chairs. The immediate worry is that the metaphysical-nihilist has to deny Quine's criterion too because we need $\mathrm{D}^{\mathrm{v}}$, here understood as discourse about chairs, and the first-order reconstrual $\left(\mathrm{D}^{\mathrm{f}}\right)$ of $\mathrm{D}^{\mathrm{v}}$ has chair-commitments, codified by apparently true statements like $(\exists x) \mathrm{C} x$-("there are chairs"). This is an indispensability argument. Without an alternative item as useful as $\mathrm{D}^{\mathrm{v}}$, Quinean discourse-ethics commit speakers to chairs. I'll assume (along with most of the metaphysics literature) that if one restricts oneself to first-order standard quantifiers ("singular first-order quantifiers") with standard (Tarskian) semantics, one can't avoid the chair-commitments that metaphysical nihilists don't want.

Plural quantifiers to the rescue. We have an alternative plural-quantifier reconstrual of $\mathrm{D}^{\mathrm{v}},(\exists x x) \mathrm{C}^{*} x x$, where $\mathrm{C}^{*}$ applied to a plural variable means: "arranged chairlike." Given plural quantifers, we can restrict the range of our (plural) quantifiers to simples: we don't have (or need) a commitment to chairs, so it seems, because "Arrangedchairlike" enables plural-quantified sentences to do the work whenever, if we restricted ourselves to first-order quantifiers, we would instead need statements like $(\exists x) \mathrm{C} x-$ "there is a chair." Wherever speakers, restricted to first-order singular quantifiers, have to describe there being a chair, speakers who utilizes first-order plural quantifiers need only be committed to the simples that compose that chair, saying instead, "there are simples arranged chairwise." The idea, therefore, is that restricting the range of the plural quantifier variables to simples suffices for ordinary talk of artifacts of all sorts.

\footnotetext{
Footnote 25 continued

modal formalisms are already widely taken to introduce substantial additional ontology. Those philosophers, however, who think that primitive modal idioms (coupled with Quine's criterion) enables an escape from unappealing ontological commitments can be challenged by the model-truncation method just as proponents of higher-order logics are challenged. To treat modal idioms as primitives is to take them as part of logic. Equivalence, therefore, is a legitimate demand proponents of both primitive modal idioms and higher-order logics must meet, provided these proponents accept Quine's criterion. I say more about this in Sect. 7.

26 I draw material from van Inwagen (1990, 2001), and from some of the subsequent literature on this topic, e.g., Bennett (2009); Hudson (2001); Sider (2011); Wasserman (2013).
} 
And the metaphysical nihilist is vindicated — at least as far as Quine's criterion is concerned. ${ }^{27}$

Consider a pluralist language that's equi-interpretable with monadic second-order logic. ${ }^{28}$ Describe monadic second-order logic, therefore, as notationally equivalent to the appropriate plural-language formalisms. ${ }^{29}$ Also accept, from Sect. 3, the truncation-model method of constructing notational equivalents. This imports the commitments imposed by the model theory of $(2 \mathrm{~m})$ truncated logic to interpreted plurallanguage statements. Consider, therefore, a bowl of Cheerios, and recall Boolos (1984, p. 72)—here repeated:

There are, of course, quite a lot of Cheerios in the bowl, well over two hundred of them. But is there, in addition to the Cheerios, also a set of them all? And what about the $>10^{60}$ subsets of that set? (And don't forget the sets of sets of Cheerios in the bowl.) It is haywire to think that when you have some Cheerios, you are eating a set-what you're doing is: eating THE CHEERIOS. Maybe there are some reasons for thinking there is such a set-there are, after all, $>10^{60}$ ways to divide the Cheerios into two portions-but it doesn't follow just from the fact that there are some Cheerios in the bowl that, as some who theorize about the semantics of plural would have it, there is also a set of them all.

We can now see why this is misleading. No $(2 m)$ truncated model forces a commitment to sets of sets of anything. The powerset of a specific domain is one thing; the settheoretical hierarchy another. But still, does one eat a set of Cheerios? Does one run from sets? Does one put sets to flight?

For that matter, does $(2 \mathrm{~m})$ truncated model theory for monadic second-order logic require sets at all? Of course not. Something is in the range of " $\mathrm{S}$ " in each $(2 \mathrm{~m})$ truncated model, and that something bears the right counter-relation ("E") to the items plurally quantified over; but nothing requires these things be sets, or that the counter-relation be the inverse of set membership.

What else can they be? Well, whatever sorts of things that individual wolves, individual grapes, etc., can be parts of, belong to, be part of the composition of, or whatever.

\footnotetext{
27 Much of the literature, e.g., Unger (e.g., 1980), avoids discussing Quine's criterion and whether a nihilist metaphysical position is compatible with it. van Inwagen (1990) is concerned with Quine's criterion, but uses plural quantification (on the basis of Lewis' authority) while describing his meta-ontological position (2001, pp. 3-4) so: "It is of the essence of my philosophy of quantification that first-order quantification has no rivals and might just as well be called simply 'quantification'." Van Inwagen deviates from Lewis, however: in the passage the previous quotation is from, he accepts Quine's characterization of higher-order logic as (in van Inwagen's recharacterization) "attribute theory in sheep's clothing," as well as accepting, in van Inwagen (1998), Quine's purely first-order constraints. Van Inwagen's position faces insurmountable technical problems because of the equi-interpretability of monadic second-order logic with certain pluralquantification formalisms. See what follows.

28 See Boolos (1984, 1985); Linnebo (2012), especially section 2.1.

29 Lewis (1991, pp. 70-71), notably, doesn't challenge this identification, as a cursory reading might seem to imply. He resists it only on the grounds that the identification will mislead (i) if someone thinks second-order logic is disguised set theory (or property theory) because the identification will infect one's view of plural quantification as well, (ii) if someone falsely suggests plural quantification can be generalized the way higher-order logic can, (iii) if someone falsely suggests polyadic plural quantification is equally ontologically undemanding, and because (iv) it results in ugly English. None of these considerations bear on the discussion here.
} 
Maybe I can't be pursued by a set of wolves (because "set" after all, is coined terminology), but I can certainly be pursued by a bunch of wolves, an aggregate of wolves, or a lot of wolves. Furthermore, although I may not be particularly scared of any of the individual wolves, I can certainly be scared (to death) of a pack of them. Maybe packs are mereological sums. Maybe they're "groups." They're certainly something over and above the individual items in them, that's not being denied_-and that's really the point in debate about the ontological innocence of plural quantifiers. But only by trivializing the word "set" can they be glibly characterized as sets.

So the singularist - as Lewis calls the opponent of the ontological innocence of plural quantification - needn't think that heaps of Cheerios (as in, 'I'm eating a heap of Cheerios") are sets. But is a heap of Cheerios, a bunch of wolves, a flock of birds anything more than the individual Cheerios, the individual wolves, or the individual birds?

This last question betrays the fact that Boolos (and Black) rely on metaphysical intuitions that conflict with Quine's ethics of ontological commitment, and that Boolos — at least—is committed to. Any proponent of Quine's criterion should respond this way: Yes, I share your intuitions that, metaphysically speaking, there is nothing in that bowl of Cheerios except for individual Cheerios. I don't intuit (or see) a mereological sum or bunch of Cheerios except insofar as I see a lot of Cheerios hanging out together in a bowl. But those intuitions are irrelevant, just as irrelevant as when I say that "There are Greek goddesses who are as prominent as Greek gods," I have the accompanying intuition that there are no Greek gods and goddesses. What's relevant aren't these intuitions but what I need to say.

The truncation-model method shows that by employing plural quantification, I'm saying (in a notationally-disguised) way that not only are there Cheerios but something else: a bunch of them. And it's my ability to talk about not only the particular Cheerios but also the lot (or bunch or aggregate) of them that enables the increased expressive power of plural quantification.

Here is another illustration of this important point. Higginbotham (2000, p. 85) writes:

We can instead regard [ $(2 \mathrm{~m})$ truncated model theory] as merely giving a picture, within a first-order language, of what is intended, and deny that the values of predicate variables are objects, construing them instead, as Frege did, as essentially predicational.

One can, indeed, do this. But that's deserting the Quinean ethics of commitment. The point, again, is what you have to say. If you deem what you have to say as not directed to an object, that's imposing a foreign criterion of ontological commitment (really — a foreign criterion for what exists), and deserting the Quinean one. I stress again the game in play here: Acceptance of Quine's criterion is the fundamental ground-rule, one accepted by Quine (of course) but also by the many opponents of the strict Quinean criterion. ${ }^{30}$

Given Quine's criterion, what commits you is what you have to say. If you say something about the fusion (or bunch or aggregate or whatever) of Cheerios in addition

30 See the end of Sect. 8 for the possibility of changing the game: deserting Quine's criterion. 
to what you say about each and all of the Cheerios then you are committed to the bunch or aggregate or fusion or whatever of Cheerios as well. ${ }^{31}$

It's surprising how often philosophers-especially in philosophy of logic or metaphysics — want something for nothing. But logic forbids this. Plural quantifiers import increased expressive strength in palpable ways: evading indispensability arguments is one of those. (That's why plural quantifiers are popular with metaphysicians.) Why isn't it obvious-I really want to ask this - that how this expressive strength is procured is technical, in the sense that what strictly goes beyond first-order resources can be made explicit? And why isn't it obvious that once this is made explicit, the result most likely begs the question against the (strict Quinean) opponent?

\section{Lewis on everything}

Lewis (1991, pp. 66-68) runs an interesting argument for plural quantification, based on the "singularist" attempting to interpret what the "pluralist" says. The reinterpretations vigorously sprint up a set-structural hierarchy (moving from sets to proper classes to "awesome classes," to, etc., ...). Lewis (1991, p. 68) writes:

But let's cut a long story short. Whatever class-like things there may be together, holding none in reserve, it seems we can truly say that there are those of them that are non-self-members. Maybe the singularist replies that some mystical censor stops us from quantifying over absolutely everything without restriction. Lo, he violates his own stricture in the very act of proclaiming it! (Italics his)

Lewis adds:

We embrace plural quantification over all the things there are. (Italics his)

The claim is that there is an interpretation of a plural-quantification formalism with everything as its domain. Can we apply the truncation-model method to this interpretation? Doing so requires an item that appears twice: in the extension of " $S$ " and in the anti-extension of "S"!

What follows? Well, it isn't as obvious as Lewis makes it out to be. ${ }^{32}$ Can we plurally quantify over all the things there are? Are we simultaneously unable to singularly quantify over all the things there are? To begin with, Quine (e.g., 1986) would demur.

\footnotetext{
31 The Quinean criterion doesn't require there be a natural or uniform or even easy way to characterize in the vernacular the additional commitments plural quantification harbors. For example, there might not be a simple phrase "bunch," "group," "aggregate," etc., that's always available to characterize what kind of thing the vernacular plural-quantifiers induce commitments to. We're committed all the same according to the Quinean ethics of ontological commitment. (My thanks to one of the referees for pressing me about this.)

Notice, despite this, that even commonsensically we recognize that bunches of things do stuff that individuals $d o n$ 't do. For example, those apples weighing two pounds isn't each and every one of those apples weighing two pounds. (My thanks to a referee for this particular example of the point.)

32 A substantial literature exists on "Absolute Generality" that makes this clear. See Rayo and Uzquiano (2006) for pertinent articles, as well as earlier cited articles, e.g., McGee (2000) and Williamson (2003). My purposes in bringing up this interesting literature are strictly restricted to evaluating potential threats to the truncation-model method.
} 
For his commitments to sets, in particular, goes only so far as the needs of applied mathematics do, and that involves very little of the set-theoretic hierarchy. More importantly, Quine's criterion (strict or otherwise) does not require that quantifiers range over everything. Additional arguments are needed to force the issue. ${ }^{33}$ I won't pursue this further because philosophers differ with Quine (and each other) on substantial issues, such as these, while still accepting his criterion-while still accepting the strict criterion. This paper is concerned—as much as possible—only with philosophers who accept Quine's criterion, while dropping Quine's demand that this criterion be applied only within first-order formalisms.

There is one important point to make about everything, however. It might seem a flaw is exposed in truncation-model constructions if we can plurally quantify over all the things that there are, but can't singularly quantify over them. But this is the wrong conclusion. Using paradoxical reasoning, to show the domain of everything can be plurally quantified over but not singularly quantified over, doesn't show that the truncation-model method is flawed. It just shows something already established: plural quantification masks ontological commitments. The conclusion to draw, therefore, is that in using plural quantifiers to range over "everything" we simply evade explicit paradox by forbidding explicit expression (in adopting pluralist formalisms) of additional ontological commitments that we (nevertheless) incur by plurally quantifying over what we (falsely) take to be everything.

\section{Primitive logical idioms and burdens of proof}

Unlike Quine's (widely rejected) first-order rendition technique which broadly applies (because it doesn't respect the metalogical properties of alternative logics), the truncation-model method looks more demanding because it isn't technically transparent when it can and can't be applied. Consider modal logics. Some philosophers think the modal idioms are logical primitives that therefore have the same effect on ontological commitments that many similarly urge plural quantification to have: none whatsoever. $^{34}$

Suppose that-success with higher-order logics notwithstanding-some modal formalism MF (with a semantics) seems resistant to the truncation-model recipe. ${ }^{35}$ And suppose there are philosophical proponents of this formalism who claim that taking MF idioms as primitives evades undesirable ontological commitments while enabling desirable expressive strengths. Suppose we use the semantics for MF to generate a rendition of MF in an interpreted first-order formalism. Polemically, unfortunately,

\footnotetext{
33 For example, that (Williamson 2003, and others) the interpretation of negative existentials requires their quantifiers range over everything, or (Linnebo 2006), "the semantic argument," that a scientific and systematic semantics can't be open-ended and essentially incomplete. The pure Quinean rejects the latter claim. (I think he can also reject the former.) Every science is incomplete - to a Quinean. Why is semantics any different? To pursue this debate further, however, is to tussle over topics outside the scope of this paper. 34 E.g., Field (1989). Notably, David Lewis does not have this view about modal formalisms-he instead uses the standard truth-conditions for modal logic to induce first-order renditions of modal language that are in turn evaluated for ontological commitments in just the way Quine's approach evaluates them.
}

35 Contrary to my suggestion in footnote 25 . 
this leaves the opponent of MF in exactly the same position vis-à-vis MF as Quine himself is vis-à-vis Boolos-as described in Sect. 2. MF appears to be logic exactly the way that higher-order logic appears to be logic. A rendition of it in an interpreted first-order formalism perhaps distorts its metalogical properties. Is the proponent of Quine's strict criterion without a response?

No. The proponent can argue this way: Natural-language intuitions about when an idiom does or doesn't involve ontological commitment can't be trusted (as shown by the success of the of the truncation-model method applied to monadic second-order logic). We know, therefore, that non-first-order logical idioms can secretively harbor ontological commitments undiscovered until notational equivalents reveal them. Where does this leave us with respect to MF? In philosophical limbo. If non-firstorder logical idioms have additional expressive strengths, they can't be trusted until proven innocent. The Quinean, thus, shouldn't deny that these idioms are innocent of additional commitments; he should deny that he (or anyone else) knows whether they are ontologically innocent or not.

Notice this "argument from ignorance" suffices to strip the rationale from programs that try to escape ontological commitments by using primitive non-first-order logical idioms. The burden is on the proponent of such programs to show their ontological innocence. ${ }^{36}$ One last point. Quine himself (e.g., 1974) took substitutional quantification, when it could be implemented, as ontologically innocent. And many philosophers have agreed. The proponent of Quine's strict criterion can take a harder line about this than Quine did. Without a notationally-equivalent construction from a first-order language coupled with objectual model-theoretic semantics, the appropriate attitude to take towards the ontological implications of substitutional quantification is also one of ignorance.

\section{Some conclusions}

There are several non-first-order idioms philosophers have adopted-in one or another quest to escape the first-order straitjacket-arguing (i) that the new ideology isn't relevant to ontological commitment while simultaneously (ii) luxuriating in its suspiciously-rich expressive strength. The quotation from Boolos lists only some of these non-first-order idioms. In some cases, as I've indicated, it's easy to construct notational equivalents of these that straightforwardly show in what ways their expressive strength and ontological commitments outrun first-order resources. The results, invariably, aren't good news for proponents: the formalisms are not ontologically innocent. If there are cases, however, where the truncation-model method hasn't been shown to apply, the debating situation is delicate. If the proponent of the non-first-order formalism (foolishly) accepts Quine's first-order rendition method, then the formalism can still be convicted of importing additional ontological commitments because of the additional nonlogical structure of the interpreted first-order theory that the formalism

\footnotetext{
36 Recall the beginning of Sect. 2: the proponent of Quine's criterion, too, must explain why the predicates and the logical sentential connectives of any interpreted first-order theory are irrelevant to ontology. That can't be presumed.
} 
is rendered in. If, however, the proponent insists the formalism is an alternative logic, as do proponents of modal logic (or substitutional quantification) who insist that, similarly to other logical idioms, the additional idioms are primitives, then the proponent of Quine's strict criterion need only point out that primitive logical idioms can harbor ontological commitments-especially if additional expressive strength results. Ignorance about ontological commitments is construed against the opponent of Quine's strict criterion.

I end this paper with a more radical suggestion, however. The metaphysical intuitions of Black and Boolos about bowls of Cheerios, packs of wolves, and the like, mentioned in Sect. 5, reveal that we all have strong metaphysical intuitions about what things there are that come apart from the dictates of Quinean syntactic ethics. So, I claim, Boolos and Lewis inadvertently make the right point: We can plurally quantify over Cheerios or wolves or whatever even if there are no sets or fusions or any other sort of "plural" entity that the Cheerios or wolves or whatever makes up. But it must be added: And we can singularly quantify without objects! (Just as we do with goddesses and gods and dreams figures and all other sorts of whatnot.) But this radical suggestion—rejecting Quine's criterion entirely—must be explored elsewhere. ${ }^{37}$

Acknowledgements My gratitude to the four anonymous referees of this paper for thoughtful and probing criticisms that helped improve this paper a great deal. My especial thanks for their persistence in forcing me to expand my exposition of some of the trickier maneuvers in the paper-technical and otherwise. My thanks to Otávio Bueno and Gila Sher for inviting my contribution to this special issue.

Open Access This article is distributed under the terms of the Creative Commons Attribution License which permits any use, distribution, and reproduction in any medium, provided the original author(s) and the source are credited.

\section{References}

Alston, W. P. (1958). Ontological commitment. Philosophical Studies, 9, 8-17.

Azzouni, J. (1994). Metaphysical myths, mathematical practice: The epistemology and the ontology of the exact sciences. Cambridge: Cambridge University Press.

Azzouni, J. (1997). Applied mathematics, existential commitment and the Quine-Putnam indispensability thesis. Philosophia Mathematica, 5(3), 193-209.

Azzouni, J. (2004). Deflating existential consequence: A case for nominalism. Oxford: Oxford University Press.

Azzouni, J. (2010). Talking about nothing: Numbers, hallucinations, and fictions. Oxford: Oxford University Press.

Bennett, K. (2009). Composition, collocation, and metaontology. In D. J. Chalmers, D. Manley, \& R. Wasserman (Eds.), Metametaphysics: New essays on the foundations of ontology (pp. 38-76). Oxford: Oxford University Press.

Black, M. (1971). The elusiveness of sets. Review of metaphysics, 24, 614-626.

Boolos, G. (1998). On second-order logic. In R. C. Jeffrey (Ed.), Logic, logic, and logic (pp. 37-53). Harvard: Harvard University Press.

Boolos, G. (1984). To be is to be a value of a variable (or to be some values of some variables). In R. C. Jeffrey (Ed.), Logic, logic, and logic (pp. 54-72). Harvard: Harvard University Press.

Boolos, G. (1985). Nominalist platonism. In R. C. Jeffrey (Ed.), Logic, logic, and logic (pp. 73-87). Harvard: Harvard University Press.

37 See, in particular, Azzouni (1997, 2004, 2010). 
Field, H. (1980). Science without numbers: A defence of nominalism. Princeton, New Jersey: Princeton University Press.

Field, H. (1989). Realism, mathematics and modality. Oxford: Basil Blackwell.

Halmos, P. R. (1960). Naive set theory. New York: Van Nostrand Reinhold Company.

Higginbotham, J. (2000). On second-order logic and natural language. In Gila Sher \& Richard Tieszen (Eds.), Between logic and intuition: Essays in honor of Charles Parsons (pp. 79-99). Cambridge: Cambridge University Press.

Hudson, H. (2001). A materialist metaphysics of the human person. Ithaca: Cornell University Press.

Lewis, D. (1991). Parts of classes. Oxford: Blackwell.

Linnebo, Ø. (2006). Sets, properties, and unrestricted quantification. In Rayo \& Uzquiano (Eds.), Absolute generality (pp. 149-178). Oxford: Oxford University Press.

Linnebo, Ø. (2012). Plural quantification. In Edward N. Zalta (Ed.), Stanford encyclopedia of philosophy. Stanford, California: CSLI. http://plato.stanford.edu/.

McGee, V. (2000). Everything. In G. Sher \& R. Tiezen (Eds.), Between logic and intuition: Essays in honor of Charles Parsons. Cambridge: Cambridge University Press.

Quine, W. V. O. (1953). On what there is. From a logical point of view (1980) (2nd ed., pp. 1-19). Cambridge, Massachusetts: Harvard University Press.

Quine, W. V. O. (1970). Philosophy of logic. Englewood Cliffs, NJ: Prentice Hall.

Quine, W. V. O. (1974). The roots of reference. La Salle, Illinois: Open Court Publishing.

Quine, W. V. O. (1986). Reply to Charles Parsons. In Hahn \& Schilpp (Eds.), The philosophy of W.V. Quine (pp. 396-403). La Salle, Illinois: Open Court Publishing.

Rayo, A., \& Uzquiano, G. (2006). Absolute generality. Oxford: Oxford University Press.

Shapiro, S. (1985). Second-order languages and mathematical practice. Journal of Symbolic Logic, 50, $714-742$.

Shapiro, S. (1997). Philosophy of mathematics: Stucture and ontology. Oxford: Oxford University Press.

Shapiro, S. (1999). Do not claim too much: Second-order logic and first-order logic. Philosophia Mathematica, 7, 42-64.

Shapiro, S. (2012). Higher-order logic or set theory: A false dilemma. Philosophia Mathematica, 20, 305323.

Sider, T. (2011). Writing the book of the world. Oxford: Oxford University Press.

Unger, P. (1980). The problem of the many. In J. O. Urmson \& G. J. Warnock (Eds.), Philosophical papers (pp. 113-182). Oxford: Oxford University Press.

van Benthem, J., \& Kees, D. (1983). Higher-order logic. In D. Gabbay \& F. Guenthner (Eds.), Handbook of philosophical logic (Vol. 1, pp. 275-330). Dordrecht: Reidel.

van Inwagen, J. (1990). Material beings. Ithaca, NY: Cornell University Press.

van Inwagen, P. (1998). Meta-ontology. In Ontology, identity, and modality (pp. 13-31). Cambridge: Cambridge University Press (2001).

van Inwagen, P. (2001). Introduction. In Ontology, identity, and modality (pp. 1-10). Cambridge: Cambridge University Press.

Wasserman, R. (2013). Material constitution. In Edward N. Zalta (Ed.), Stanford encyclopedia of philosophy. Stanford, California: CSLI. http://plato.stanford.edu/.

Williamson, T. (2003). Everything. Philosophical Perspectives, 17, 415-465. 